spatial mode, we set

$$
2\left(\frac{1}{\delta x^{2}}+\frac{1}{\delta y^{2}}+\frac{1}{\delta z^{2}}\right)^{1 / 2} \leq \frac{2}{\delta t} .
$$

The algorithm stability condition follows immediately from (24). In an inhomogeneous region of space, it is difficult to determine a spectrum of $\lambda$ analogous to (23) for all possible lattice spatial modes. For absolute algorithm stability, (7) suffices because it represents a "worst case" choice of $\delta t$.

\section{ACKNOWLEDGMENT}

The authors wish to thank Dr. G. O. Roberts of the Engineering Sciences Department of Northwestern University for his useful comments on algorithm stability.

\section{REFERENCES}

[1] T. K. Wu and L. L. Tsai, "Numerical analysis of electromagnetic fields in biological tissues," Proc. IEEE (Lett.), vol. 62, pp. 1167-1168, Aug. 1974.

[2] R. F. Harrington, Field Computation by Moment Methods. New York: Macmillan, 1968, ch. 3.

[3] B. H. McDonald and A. Wexler, "Finite-element solution of unbounded field problems," IEEE Trans. Microwave Theory Tech. (197\% Symp. Issue), vol. MTT-20, pp. 841-847, Dec. 1972.

[4] D. R. Wilton and R. Mittra, "A new numerical approach to the calculation of electromagnetic scattering properties of twodimensional bodies of arbitrary cross section," IEEE Trans. Antennas Propagat., vol. AP-20, pp. 310-317, May 1972.

[5] Vogelback Computing Center, Northwestern Univ., Evanston, Ill., Library no. NUCC288, Subroutine LUECS.

[6] K. S. Yee, "Numerical solution of initial boundary value problems involving Maxwell's equations in isotropic media," IEEE Trans. Antennas Propagat., vol. AP-14, pp. 302-307, May 1966.

[7] D. S. Jones, The Theory of Electromagnetism. New York: Macmillan, 1964, pp. 450-452.

\title{
Worst Case Network Tolerance Optimization
}

\author{
JOHN W. BANDLER, SENIOR MEMBER, IEEE, PETER C. LIU, STUDENT MEMBER, IEEE, AND \\ JAMES H. K. CHEN, STUDENT MEMBER, IEEE
}

\begin{abstract}
The theory and its implementation in a new useroriented computer program package is described for solving continuous or discrete worst case tolerance assignment problems simultaneously with the selection of the most favorable nominal design. Basically, the tolerance problem is to ensure that a design subject to specified tolerances will meet performance or other specifications. Our approach, which is believed to be new to the microwave design area, can solve a variety of tolerance and related problems. Dakin's tree search, a new quasi-Newton minimization method, and least $p$ th approximation are used. The program itself is organized such that future additions and deletions of performance specifications and constraints, and replacement of cost functions and optimization methods are readily realized. Options and default values are used to enhance flexibility. The full Fortran listing of the program and documentation will be made available.
\end{abstract}

Manuscript received August 5, 1974; revised February 3, 1975. This work was supported by the National Research Council of Canada in part under Grant A 7239, and in part by a scholarship to J. H. K. Chen.

J. W. Bandler is with the Group on Simulation, Optimization, and Control and the Department of Electrical Engineering, McMaster University Hamilton, Ont., Canada.

P. C. Liu was with the Department of Electrical Engineering, McMaster University, Hamilton, Ont., Canada. He is now with Bell-Northern Research, Verdun, P. Q., Canada.

J. H. K. Chen was with McMaster University, Hamilton, Ont., Canada. He is now with Bell-Northern Research, Ottawa, Ont., Canada.

\section{INTRODUCTION}

A NEW user-oriented computer program package called TOLOPT (TOLerance optimization) is presented which can solve continuous or discrete worst case tolerance assignment problems simultaneously with the selection of the most favorable nominal design, taking full advantage of the most recent developments in optimization practice. Our approach, it is believed, is new to the microwave design area. Previous design work has usually been concentrated on obtaining a best nominal design, disregarding the manufacturing tolerances and material uncertainties. Basically, the tolerance assignment problem is to ensure that a design, when fabricated, will meet performance or other specifications.

The package is designed to handle the objective functions, performance specifications, and parameter constraints in a unified manner such that any of the nominal values or tolerances (relative or absolute) can be fixed or varied automatically at the user's discretion. Time-saving techniques for choosing constraints (vertices selection) are incorporated. The routine involved also checks assump- 
tions and performs worst case analyses. The paper also contains a brief discussion of network symmetry and how it may be implemented to further reduce the number of constraints.

The continuous and (optional) discrete optimization methods are programmed in such a way that they may be used as a separate unit. This part, called DIsop2 and incorporating several optional features, is an updated version of DIsOPT, which has been applied successfully in many different areas [1]-[3]. Dakin's tree search for discrete problems [4], efficient gradient minimization of functions of many variables by a recent quasi-Newton method [5], and the latest developments in least $p$ th approximation by Bandler and Charalambous [6]-[9] are employed. Extrapolation is also featured [10].

Another practical problem which is analogous to the tolerance assignment problem is to determine the optimum component values to a certain number of significant figures, which can be done with DIsop2.

The ToLop program is organized in such a way that future additions and deletions of performance specifications and constraints, and replacement of cost functions and optimization methods are readily realized. Any of the two different vertices elimination schemes can be bypassed or replaced by the user. It is felt that the program is particularly flexible in the way that the user may enter at any stage of the problem's solution. The user supplies the network analysis subroutines. With an arbitrary initial acceptable or unacceptable design as a starting point, the program would output the set of nominal component parameters together with a set of optimal tolerances satisfying all the specifications in the worst case sense. The user decides on a continuous solution and/or discrete solutions.

The package, written in Fortran IV and run on a CDC 6400 digital computer, will be made available. Several test examples are presented here to illustrate the theory and practice of the approach.

\section{THE TOLERANCE PROBLEM}

\section{Introduction $[11]-[15]$}

A design consists of design data of the nominal design point $\phi^{0} \triangleq\left[\phi_{1}{ }^{0} \phi_{2}{ }^{0} \cdots \phi_{k}{ }^{0}\right]^{T}$ and a set of associated tolerances $\varepsilon \triangleq\left[\epsilon_{1} \epsilon_{2} \cdots \epsilon_{k}\right]^{T}$, where $k$ is the number of network parameters. Let $I_{\phi} \triangleq\{1,2, \cdots, k\}$ be the index set for these parameters. We take the $i$ th absolute tolerance as $\epsilon_{i}$ in the discussion in this section; however, the discussion applies also to the relative tolerance $t_{i} \triangleq \epsilon_{i} / \phi_{i}{ }^{0}$ without any conceptual difference. An outcome of a circuit is any point $\phi \triangleq\left[\phi_{1} \phi_{2} \cdots \phi_{k}\right]^{T}$ in the tolerance region $R_{t} \triangleq$ $\left\{\phi \mid \phi_{i}{ }^{0}-\epsilon_{i} \leq \phi_{i} \leq \phi_{i}{ }^{0}+\epsilon_{i}, i \in I_{\phi}\right\}$. The constraint region $R_{c}$ is the region of points $\phi$ such that all performance specifications and constraints are satisfied by the circuit. The worst case design requires that $R_{t} \subseteq R_{c}$. The optimal worst case design can, therefore, be stated as: minimize some cost function $C$ subject to $R_{t} \subseteq R_{c}$.

We need the following assumptions on $R_{c}$ in order to make the problem tractable.

\section{Assumptions on $R_{c}$}

1) $R_{c}$ is not empty.

2) $R_{c}$ is bounded and simply connected.

3) $R_{c}$ is at least one-dimensionally convex.

Assumption 1) guarantees there is at least one feasible solution, and assumption 2) is a computational safeguard against infinite parameter values.

We say that $R_{c}$ is one-dimensionally corvex if for all $j \in I_{\phi}[11]$

$$
\phi^{a}, \phi^{b(j)} \triangleq \phi^{a}+\alpha u_{j} \in R_{c}
$$

where $\alpha$ is some constant and $\boldsymbol{u}_{j}$ is the $j$ th unit vector, implies that

$$
\phi=\phi^{a}+\lambda\left(\phi^{b(j)}-\phi^{a}\right) \in R_{c}
$$

for all $0 \leq \lambda \leq 1$.

Let us also define the set of vertices $R_{v} \triangleq\left\{\phi^{1}, \phi^{2}, \cdots, \phi^{2^{k}}\right\}$, and the corresponding index set $I_{v}$, where

$$
\phi^{r} \triangleq \phi^{0}+E \mu(r)
$$

$\mu_{j}(r) \in\{-1,1\}$ and satisfies the relation

$$
r=1+\sum_{j=1}^{k}\left(\frac{\mu_{j}(r)+1}{2}\right) 2^{j-1}
$$

where $E$ is a diagonal matrix with $\epsilon_{i}$ as the $i$ th element. Under the foregoing assumptions

$$
R_{v} \subseteq R_{c} \Rightarrow R_{t} \subseteq R_{c}
$$

See [11] for the proof, and Fig. 1 for an illustration of the concepts.

\section{Assumptions on the Constraints}

$R_{c}$ may be defined specifically by a set of constraint functions, namely,

$$
R_{c} \triangleq\left\{\phi \mid g_{i}(\phi) \geq 0, i \in I_{c}\right\}
$$

where $I_{c}$ is the index set for the functions. Concave constraint functions or, more generally, quasi-concave functions will satisfy assumption 3$)$. The function $g(\phi)$ (dropping the subscript $i, i \in I_{c}$ ) is said to be quasiconcave in a region if, for all $\phi^{a}, \phi^{b}$ in the region,

$$
g\left(\boldsymbol{\phi}^{a}+\lambda\left(\boldsymbol{\phi}^{b}-\boldsymbol{\phi}^{a}\right)\right) \geq \min \left[g\left(\boldsymbol{\phi}^{a}\right), g\left(\boldsymbol{\phi}^{b}\right)\right]
$$

for all $0 \leq \lambda \leq 1$. An immediate consequence of (7) is that a region defined as $\{\phi \mid g(\phi) \geq 0\}$ is convex [16]. The intersection of convex regions is also convex, and the multidimensional convexity implies the one-dimensional convexity of assumption 3 ).

If the point $\phi^{b}$ in (7) is defined as in (1), then the function $g(\phi)$ satisfying (7) will be called a one-dimensional quasi-concave function. The region defined by these functions is one-dimensionally convex. Assumption 3) is satisfied [17]. Throughout the following discussions, we will assume the functions to have this less restrictive property.

Under the foregoing assumptions we have the nonlinear programming problem: minimize $C$ subject to $g_{i}\left(\phi^{r}\right) \geq 0$ for all $\phi^{r} \in R_{v}, i \in I_{c}$. 
(a)

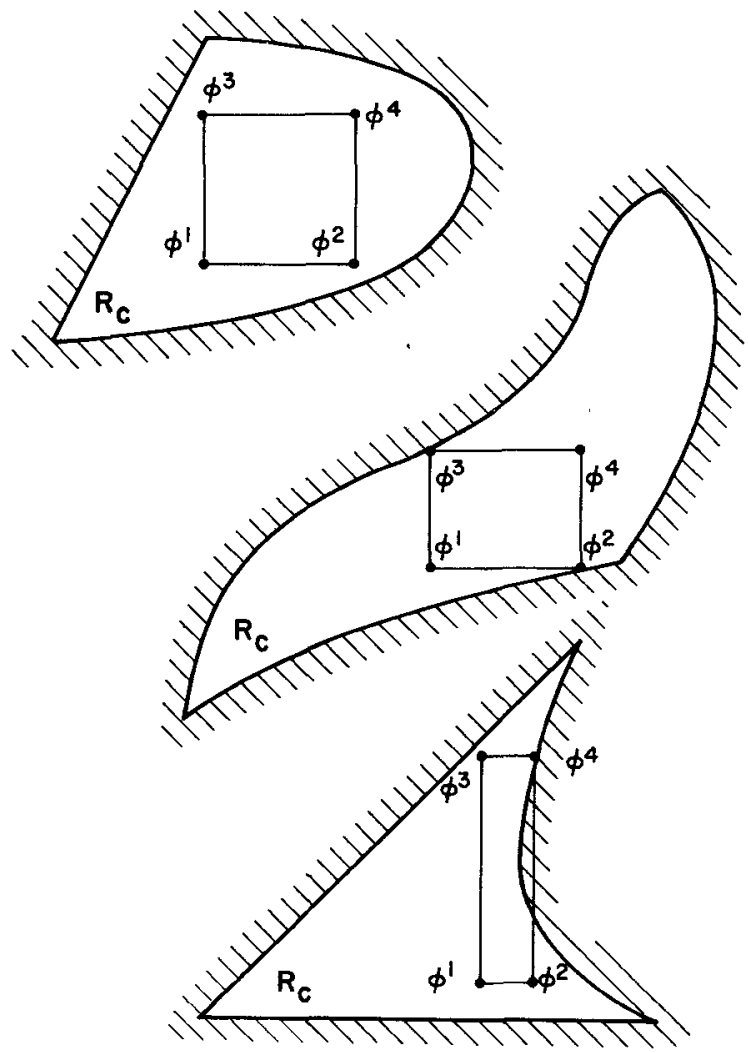

Fig. 1. Possible regions $R_{c}$. (a) $R_{v}$ is a subset of $R_{c}$ implies that $R_{t}$ is a subset of $R_{c}$. (b) $R_{v}$ is a subset of $R_{o}$ implies that $R_{t}$ is a subset of $R_{c}$. (c) $R_{v}$ is a subset of $R_{c}$ does not imply that $R_{t}$ is a subset of $R_{c}$.

\section{Conditions for Monotonicity}

Given a differentiable one-dimensional quasi-concave function $g(\phi)$ (see, for example, Fig. 2), the function is monotonic with respect to $\phi$ on an interval $\left[\phi^{a}, \phi^{b}\right]$ if $\operatorname{sgn}\left(g^{\prime}\left(\phi^{a}\right)\right)=\operatorname{sgn}\left(g^{\prime}\left(\phi^{b}\right)\right)$. Furthermore, the minimum of $g(\phi)$ is at $\phi=\frac{1}{2}\left[\phi^{a}+\phi^{b}-\operatorname{sgn}\left(g^{\prime}\left(\phi^{a}\right)\right)\left(\phi^{b}-\phi^{a}\right)\right]$. This may be proved as follows.

Consider the case $\operatorname{sgn}\left(g^{\prime}\left(\phi^{a}\right)\right)=\operatorname{sgn}\left(g^{\prime}\left(\phi^{b}\right)\right)>0$. Suppose $g(\phi)$ is not monotonic. Then there exist two points $\phi^{1}, \phi^{2} \in\left(\phi^{a}, \phi^{b}\right), \phi^{2}>\phi^{1}$ such that $g^{\prime}\left(\phi^{1}\right)<0$ and $g\left(\phi^{2}\right)>g\left(\phi^{1}\right)$. Thus $g\left(\phi^{1}+\lambda\left(\phi^{2}-\phi^{1}\right)\right)$ for some $0<$ $\lambda<1$ is smaller than $g\left(\phi^{1}\right)$, which contradicts $(7)$. The assumption that $g(\phi)$ is not monotonic is wrong, hence $g(\phi)$ is monotonic. Furthermore, it is nondecreasing on $\left[\phi^{a}, \phi^{b}\right]$. The minimum is at $\phi^{a}$.

Similarly, it may be proved that the case sgn $\left(g^{\prime}\left(\phi^{a}\right)\right)=$ sgn $\left(g^{\prime}\left(\phi^{b}\right)\right)<0$ implies monotonicity with $g(\phi)$ nonincreasing on $\left[\phi^{a}, \phi^{b}\right]$. The minimum is at $\phi^{b}$.

\section{Implications of Monotonicity}

Suppose $g_{i}$ is monotonic in the same direction with respect to $\phi_{j}$ throughout $R_{t}$. Then the minimum of $g_{i}$ is on the hyperplane $\phi_{j}=\phi_{j}{ }^{0}-\epsilon_{j} \operatorname{sgn}\left(\partial g_{i} / \partial \phi_{j}\right)$. Hence only those vertices which lie on that hyperplane need to be constrained. In general, if there are $l$ variables with respect to which the function $g_{i}$ is monotonic in this way, the $2^{k-l}$ vertices lying on the intersection of the hyperplanes are constrained. In the case where $l=k$, the vertex of minimum $g$ occurs at $\boldsymbol{\phi}^{r}$ where

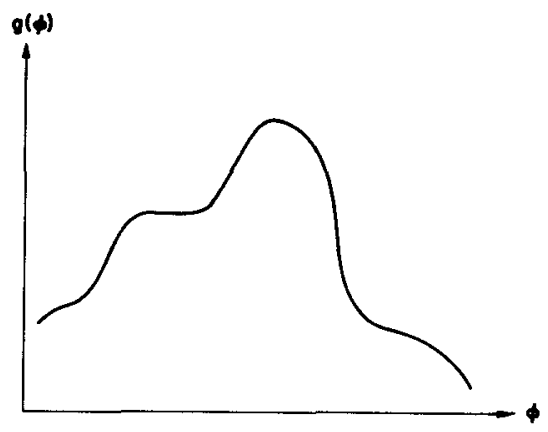

Fig. 2. A one-dimensional quasi-concave function.

$$
\phi_{j}{ }^{r}=\phi_{j}{ }^{0}-\epsilon_{j} \operatorname{sgn}\left(\frac{\partial g_{i}}{\partial \phi_{j}}\right) \text { for all } j \in I_{\phi} .
$$

Let the set that contains the critical vertices be denoted by $R_{v}^{\prime}(i) \subseteq R_{v}$. The modified problem is: minimize $C$ subject to $g_{i}\left(\boldsymbol{\phi}^{r}\right) \geq 0$, for all $\boldsymbol{\phi}^{r} \in R_{v}{ }^{\prime}(i), i \in I_{\boldsymbol{c}}$.

\section{The Vertices Elimination Schemes}

Various schemes may be developed to identify or to predict the most critical vertices that are likely to give rise to active constraints. Our proposed schemes will eliminate all but one vertex for each constraint function in the most favorable conditions. In this case, the subsequent computational effort for the optimization procedure is comparable to the linearization technique commonly used. When monotonicity assumptions are not sufficient to describe the function behavior, our scheme will increase the number of vertices until, at worst, all the $2^{k}$ vertices are included.

In principle, our schemes may be stated as follows:

Step 1) systematic generation, for positive $\alpha$, of sets of points

$$
\phi^{a}, \phi^{b(j)}=\phi^{a}+\alpha u_{j}
$$

Step 2) evaluation of the function values and the partial derivatives at these points.

Step 3) If

$$
\operatorname{sgn}\left(\left.\frac{\partial g_{i}}{\partial \phi_{j}}\right|_{\phi=\boldsymbol{\phi}^{a}}\right)=\operatorname{sgn}\left(\left.\frac{\partial g_{i}}{\partial \phi_{j}}\right|_{\dot{\phi}=\boldsymbol{\phi}^{b(j)}}\right) .
$$

eliminate the vertices $\phi^{r} \in R_{v}$ on the hyperplane

$$
\phi_{j}=\phi_{j}{ }^{0}+\epsilon_{j} \operatorname{sgn}\left(\frac{\partial g_{i}}{\partial \phi_{j}}\right) \text {. }
$$

If

$$
\operatorname{sgn}\left(\left.\frac{\partial g_{i}}{\partial \phi_{j}}\right|_{\phi=\phi^{a}}\right)<0 \text { and } \operatorname{sgn}\left(\left.\frac{\partial g_{i}}{\partial \phi_{j}}\right|_{\boldsymbol{\phi}=\boldsymbol{\phi}^{b(j)}}\right)>0
$$

note that the quasi-concavity assumption is violated.

\section{Comments}

1) We have investigated and implemented two methods for step 1), involving: a) $\phi^{a}=\phi^{0}-\epsilon_{j} u_{j}$ and $\phi^{b}=\phi^{0}+$ $\epsilon_{j} u_{j}$, for all $\left.j \in I_{\phi} ; \mathrm{b}\right)$ all the vertices of $R_{t}$. A special case which we do not consider in this paper is for $\phi^{a}=\phi^{b}$ in 
step 1), in which case the first part of step 3) is applicable. $R_{v}{ }^{\prime}(i)$ contains only one vertex.

2) It is possible to further eliminate some vertices by considering the relative magnitudes of $g_{i}\left(\boldsymbol{\phi}^{r}\right)$.

3) For method b), a worst case check can be accomplished as a by-product of the vertices elimination scheme since function values are computed at each vertex.

4) The schemes are based on local information. $R_{v}{ }^{\prime}$ should be updated at suitable intervals.

\section{Symmetry}

A circuit designer should exploit symmetry to reduce computation time. The following is an example of how it may be done in the tolerance problem.

A function is said to be symmetrical with respect to $S$ in a region if

$$
g(\boldsymbol{S} \phi)=g(\boldsymbol{\phi})
$$

where $S$ is a matrix obtained by interchanging suitable rows of a unit matrix [18]. It has exactly one entry of 1 in each row and in each column, all other entries being 0 .

A common physical symmetry configuration is a mirrorimage symmetry with respect to a center line. The $\boldsymbol{S}$ matrix in this case is

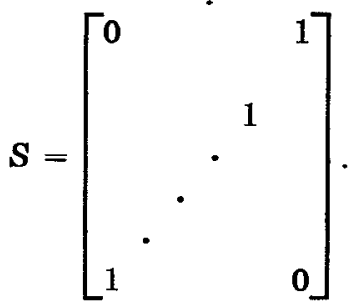

Postmultiplication of a matrix $\boldsymbol{A}$ by any $\boldsymbol{S}$ simply permutes the columns of $\boldsymbol{A}$, and premultiplication of $\boldsymbol{A}$ permutes the rows of $A . S S^{T}=1$, and $S^{T} D S=D_{s}$, where $D$ is a diagonal matrix and $D_{s}$ is also a diagonal matrix with diagonal entries permuted.

Consider symmetrical $S, \phi^{0}$, and $\varepsilon$. By this we imply

$$
\begin{aligned}
\boldsymbol{S}(\boldsymbol{S} \boldsymbol{A}) & =\boldsymbol{A} \\
\mathbf{S} \boldsymbol{\phi}^{0} & =\boldsymbol{\phi}^{0}
\end{aligned}
$$

and

$$
S^{T} E S=E .
$$

Let us premultiply the $r$ th vertex from (3) by $\mathbf{S}$, giving

$$
\begin{aligned}
S \phi^{r} & =S \phi^{0}+\boldsymbol{S}(E \mu(r)), \quad r \in I_{v} \\
& =\phi^{0}+\boldsymbol{S}\left(\boldsymbol{S}^{T} E S \boldsymbol{\mu}(r)\right) \\
& =\phi^{0}+E \boldsymbol{S} \boldsymbol{\mu}(r) .
\end{aligned}
$$

Now $S_{\mu}(r)$ is another vector with +1 and -1 entries. Let it be denoted by $\mu(s), s \in I_{v}$. In some cases $\mu(r)$ is identical to $\mu(s)$ if the vector is symmetrical. In other cases $\boldsymbol{\mu}(r) \neq \boldsymbol{\mu}(s)$. In all cases

$$
\boldsymbol{S} \boldsymbol{\phi}^{r}=\boldsymbol{\phi}^{s} .
$$

Making use of the symmetrical property of $g$

$$
g\left(\boldsymbol{S} \boldsymbol{\phi}^{r}\right)=g\left(\boldsymbol{\phi}^{r}\right)=g\left(\boldsymbol{\phi}^{s}\right) .
$$

Let the number of symmetrical vectors $\mu(r)$ and the number of pairs of nonsymmetrical $\boldsymbol{\mu}(r)$ and $\boldsymbol{\mu}(s)$ be denoted by $N(r=s)$ and $N(r \neq s)$, respectively. Then

$$
N(r=s)=2^{k-k} s, \quad 2 k_{\mathrm{s}} \leq k
$$

and

$$
N(r \neq s)=\left(2^{k}-2^{k-k_{a}}\right) / 2, \quad 2 k_{s} \leq k
$$

where $k_{s}$ is the number of pairs of symmetrical components. Therefore, there are $N(r=s)+N(r \neq s)$ effective vertices as compared to $2^{k}$ topological vertices. Take, for example, $k=6$ and $k_{s}=3$; only 36 function evaluations are required for all the 64 vertices.

The above discussion and results may be used to reduce computation time. However, in general, it is not certain that a nominal design without tolerances yielding a symmetrical solution will imply a symmetrical optimal solution with tolerances either in the continuous or in the discrete cases.

\section{OPTIMIZATION METHOISS}

\section{Nonlinear Programming Problem}

After eliminating the inactive vertices and. constraints as discussed in Section II, the tolerance problem takes the form

$$
\text { minimize } f \triangleq f(x)
$$

subject to

$$
g_{i}(x) \geq 0, \quad i=1,2, \cdots, m
$$

where $f$ is the chosen objective function (see Section IV). The vector $x$ represents a set of up to $2 k$ design variables which include the nominal values, and the relative and/or absolute tolerances of the network components. The constraint functions $g_{1}(\boldsymbol{x}), g_{2}(\boldsymbol{x}), \cdots, g_{m}(\boldsymbol{x})$ comprise the selected response specifications, component bounds, and any other constraints. The constraints are renumbered from 1 to $m$ for simplicity.

\section{Constraint Transformation}

Recently, Bandler and Charalambous have proposed a minimax approach [8] to transform a nonlinear programming problem into an unconstrained objective. The method involves minimizing the function

$V(x, \alpha)=\max _{1 \leq i \leq m}\left[f(x), f(x)-\alpha g_{i}(x)\right]$ where $\alpha>0$.

A sufficiently large value of $\alpha$ must be chosen to satisfy the inequality

$$
\frac{1}{\alpha} \sum_{i=1}^{m} u_{i}<1
$$

where the $u_{\imath}^{\prime} s$ are the Kuhn-Tucker multipliers at the optimum. This approach compares favorably with the well-regarded Fiacco-McCormick technique [19]. 
Several least $p$ th optimization algorithms are available for solving the resulting minimax problem. The function to be minimized is computed in the present paper as

$$
U(x) \leftarrow(M(x)-\epsilon)\left(\sum_{j \epsilon J}\left(\frac{e_{j}(x)-\epsilon}{M(x)-\epsilon}\right)^{q}\right)^{1 / q}
$$

where

$$
\begin{aligned}
M(x) & \leftarrow \max _{j \epsilon J} e_{j}(x) \\
\epsilon & \leftarrow\left\{\begin{array}{l}
0 \text { for } M(x) \neq 0 \\
\text { small positive number for } M(x)=0
\end{array}\right. \\
q & \leftarrow p \operatorname{sgn}(M(x)-\epsilon) \\
p & >1
\end{aligned}
$$

and if

$$
M(x)\left\{\begin{array}{l}
>0, J \leftarrow\left\{j \mid e_{j}(x)>0\right\} \\
<0, J \leftarrow\{1,2, \cdots, m+1\} .
\end{array}\right.
$$

The definition of the $e_{j}$, the appropriate value $(s)$ of $p$, and the convergence features of the algorithms are summarized in Table I (algorithms 1-4).
Another approach to nonlinear programming which utilizes a least $p$ th objective is also detailed in Table $I$ (algorithm 5). It is a modification of an existing nonparametric exterior-point algorithm described by Lootsma $[20]$.

\section{Existence of a Feasible Solution}

The existence of a feasible solution can be detected by minimizing (23) when

$$
e_{j} \leftarrow \begin{cases}-g_{j}, & j=1,2, \cdots, m \\ f-\bar{f}, & j=m+1\end{cases}
$$

where $\bar{f}$ is an upper bound on $f$. A nonpositive value of $M$ at the minimum, or even before the minimum is reached indicates that a feasible solution exists. Otherwise, no feasible solution satisfying the current set of constraints and the upper bound on the objective function value is perceivable. Only one single optimization with a small value of $p$ greater than unity is required.

\section{Unconstrained Minimization Method}

Gradient unconstrained minimization methods have become very popular because of their reported efficiency.

TABLE I

The Optional Least $p$ Th Algorithms

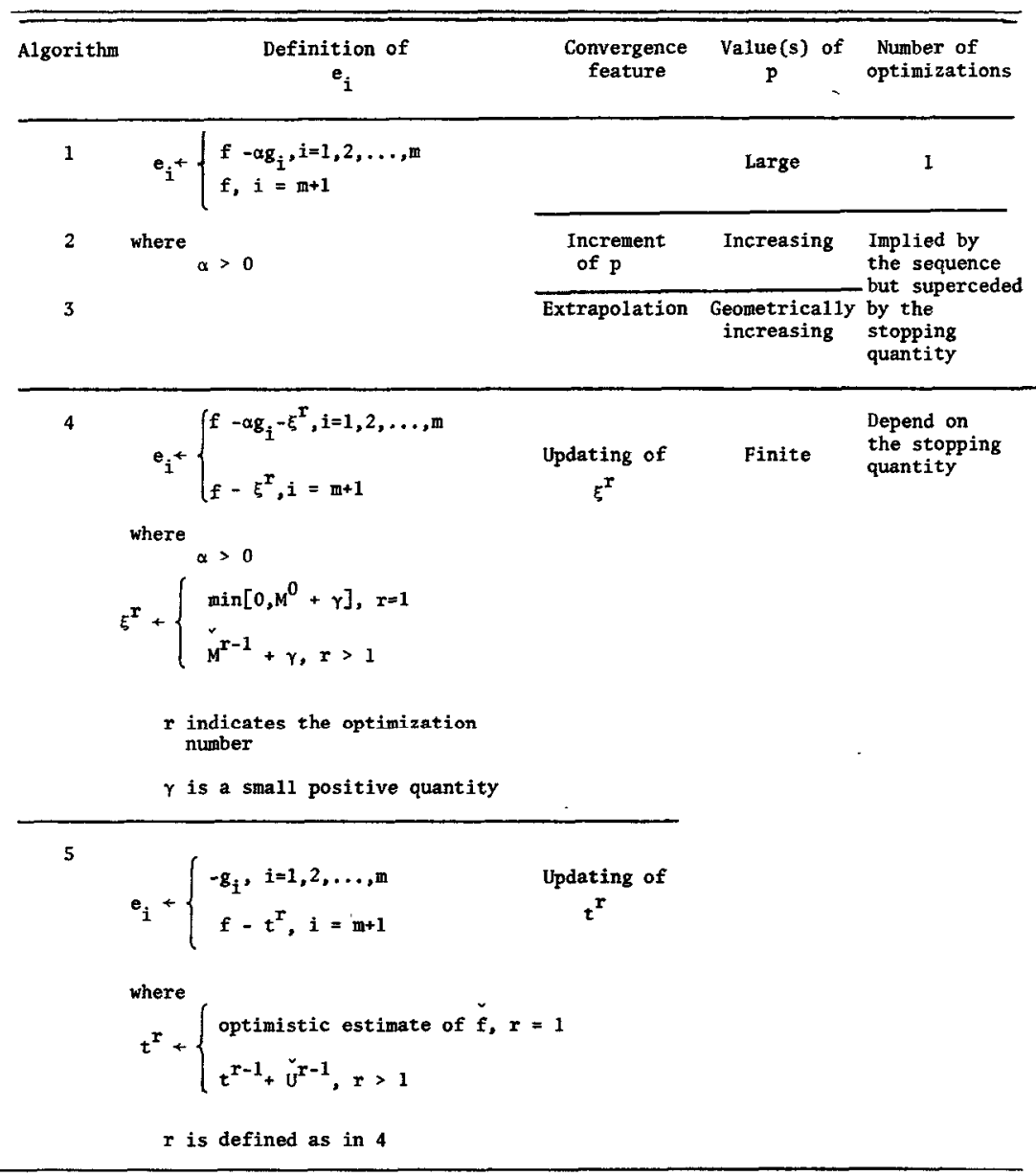


One such program is the Fortran subroutine, which utilizes first derivatives, implemented by Fletcher [5]. The method used belongs to the class of quasi-Newton methods. The direction of search $\boldsymbol{s}^{j}$ at the $j$ th iteration is calculated by solving the set of equations

$$
B^{j} \mathcal{S}^{j}=-\nabla U\left(x^{j}\right)
$$

where $\boldsymbol{B}^{j}$ is an approximation to the Hessian matrix $\boldsymbol{G}$ of $U, \nabla U$ is the gradient vector, and $x^{j}$ is the estimate of the solution at the $j$ th iteration.

As proposed by Gill and Murray [21], the matrix $B^{j}$ is factorized as

$$
B^{j}=L^{i} D^{j} L^{j T}
$$

where $L$ is a lower unit triangular matrix and $D$ is a diagonal matrix. It is important that $B^{j}$ must always be kept positive definite, and with the above factorization, it is easy to guarantee this by ensuring $d_{i i}>0$ for all $i$.

A modification of the earlier switching strategy of Fletcher [22] is used to determine the choice of the correction formula for the approximate Hessian matrix. The Davidon-Fletcher-Powell (DFP) formula is used if

$$
\delta^{T} L D L^{T} \delta<\delta^{T}\left(\nabla U\left(x^{j+1}\right)-\nabla U\left(x^{j}\right)\right)
$$

where

$$
\delta=x^{j+1}-x^{j} .
$$

Otherwise, the complementary DFP formula is used.

The minimization will be terminated when $\left|x_{i}{ }^{j+1}-x_{i}{ }^{j}\right|$ is less than a prescribed small quantity for all $i$.

\section{Discrete Optimization}

In practical design, a discrete solution may be more realistic than a continuous solution. In network toleranceoptimization problems, very often only components of certain discrete values, or having certain discrete tolerances are available on the market. At present, a general strategy for solving a nonlinear discrete programming problem is the tree-search algorithm due to Dakin [4].

Dakin's integer tree-search algorithm first finds a solution to the continuous problem. If this solution happens to be integral, the integer problem is solved. If it is not, then at least one of the integer variables, e.g., $x_{i}$, is nonintegral and assumes a value $x_{i}^{*}$, say, in this solution. The range

$$
\left[x_{i}^{*}\right]<x_{i}<\left[x_{i}^{*}\right]+1
$$

where $\left[x_{i}{ }^{*}\right]$ is the largest integer value included in $x_{i}{ }^{*}$, is inadmissible, and consequently we may divide all solutions to the given problem into two nonoverlapping groups, namely, 1) solutions in which

$$
x_{i} \leq\left[x_{i}^{*}\right]
$$

2) solutions in which

$$
x_{i} \geq\left[x_{i}^{*}\right]+1
$$

Each of the constraints is added to the continuous problem sequentially, and the corresponding augmented problems are solved. The procedure is repeated for each of the two solutions so obtained. Each resulting nonlinear programming problem thus constitutes a node, and from each node two branches may emanate. A node will be fathomed if the following happen: 1) the solution is integral; 2) no feasible solution for the current set of constraints is achievable; 3) the current optimum solution is worse than the best integer solution obtained so far. The search stops when all the nodes are fathomed.

It seems, then, that the most efficient way of searching would be to branch, at each stage, from the node with the lowest $f(x)$ value. This would minimize the searching of unlikely subtrees. To do this, all information about a node has to be retained for comparison; this may require cumbersome housekeeping and excessive storage for computer implementation. One way of compromising is to search the tree in an orderly manner; each branch is followed until it is fathomed.

The tree is not, in general, unique for a given problem. The tree structure depends on the order of partitioning on the integer variables used. The amount of computation may be vastly different for different trees.

For the case of discrete programming problems subject to uniform quantization step sizes, the Dakin algorithm is modified as follows: let $x_{i}$ be the discrete variable which assumes a nondiscrete solution $x_{i}{ }^{*}$; and let $q_{i}$ be the corresponding quantization step; then the two variable constraints added sequentially after each node become

$$
x_{2} \geq\left[x_{i}^{*} / q_{2}\right] q_{i}+q_{2}
$$

and

$$
x_{i} \leq\left[x_{i}{ }^{*} / q_{i}\right] q_{\imath} .
$$

The integer problem is thus a special case of the discrete problem with $q_{i}=1, i=1,2, \cdots, n$, where $n$ is the number of discrete variables.

If, however, a finite set of discrete values given by

$$
D_{i}=\left\{d_{i 1}, d_{i 2}, \cdots, d_{i j}, d_{i(j+1)}, \cdots, d_{i u}\right\}, \quad i=: 1,2, \cdots, n
$$

is imposed upon each of the discrete variables, the variable constraints are then added according to the following rules.

1) If

$$
d_{i j}<x_{\imath}{ }^{*}<d_{i(j+1)}
$$

then add the two constraints

$$
x_{i} \leq d_{i j}
$$

and

sequentially.

2) If

$$
x_{i} \geq d_{i(j+1)}
$$

$$
x_{i}^{*}<d_{i 1}
$$

only add the constraint

$$
x_{i} \geq d_{i 1} .
$$


3) If

$$
x_{i}^{*}>d_{i u}
$$

only add the constraint

$$
x_{i} \leq d_{i u}
$$

The resulting nonlinear programming problem at each node is solved by one of the algorithms described earlier. The feasibility check is particularly useful here since the additional variable constraints may conflict with the original constraints on the continuous problem. An upper bound $\bar{f}$, on $f(x)$, if not specified, may be taken as the current best discrete solution. For a discrete problem, the best solution among all the discrete solutions given by letting variables assume combinations of the nearest upper and lower discrete values (if they exist) may be taken as the initial upper bound on $f(x)$.

The new variable constraint added at each node excludes the preceding optimum point from the current solution space and the constraint is therefore active if the function is locally unimodal. Thus the value of the variable under the new constraint may be optionally fixed on the constraint boundary during the next optimization. See Fig. 3 for illustrations of the search procedure and a tree structure.

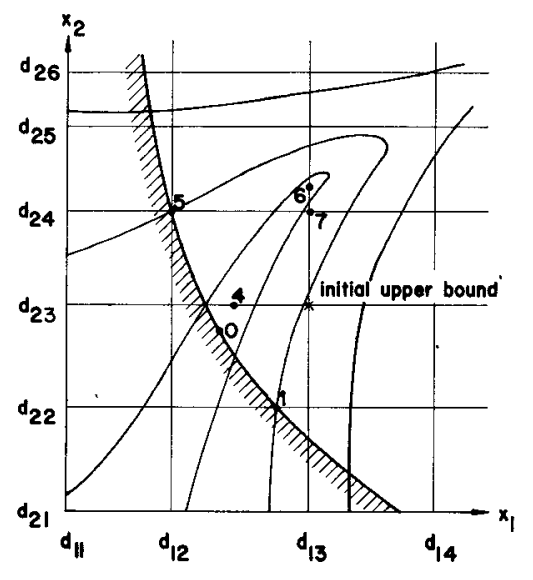

(a)

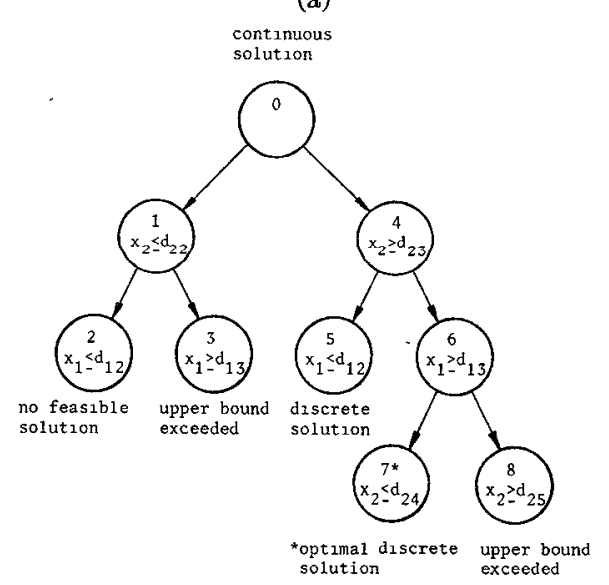

(b)

Fig. 3. An illustration of the search for discrete solutions. (a) Contours of a function of two variables with grid and intermediate solutions. (b) The tree structure.

\section{IMPLEMENTATION OF THE TOLERANCE PROBLEM}

\section{The Overall Structure of TOLOPT}

Fig. 4 displays a block diagram of the principal subprograms comprising the TOLOPT program. A brief description of these subprograms is given in this section.

TOLOPT is the subroutine called by the user. It organizes input data and coordinates other subprograms. Subroutine DIsOP2 is a general program for continuous and discrete nonlinear programming problems. Subroutine VERTST eliminates the inactive vertices of the tolerance region. Subroutine constr sets up the constraint functions based on the response specifications, component bounds, and other constraints supplied in the user subroutine USERCN. Subroutine costriv computes the cost function. The user has the option of supplying his own subroutine to define other cost functions. The user-supplied subroutine NETWRK returns the network responses and the partial derivatives.

Table II is a summary of the features and options currently incorporated in TOLOPT.

Some components of $\varepsilon$ and $\phi^{0}$ may be fixed which do not enter into the optimization parameters $x$. The user supplies the initial values of the tolerances (relative or absolute) and the nominals with an appropriate vector to indicate whether they are fixed or variable, relative or absolute. The program will assign those variable components to vector $x$.

\section{The Objective Function}

The objective function we have investigated and implemented is [11]--[13]

$$
C=\sum_{i} \frac{c_{i}}{x_{i}}
$$

where $x_{i}$ is either $\epsilon_{i}$ or $t_{i}$, and the $c_{i}$ are some suitable weighting factors supplied by the user. The default value is one. To avoid negative tolerances we let $x_{\imath}=x_{i}{ }^{2}$, where $x_{i}^{\prime}$ is taken as a new variable replacing $x_{i}$.

\section{Vertices Selection and Constraints}

Two schemes of increasing complexity are programmed in the subroutine. The user decides on the maximum allowable calls for each scheme, starting with the simple one. He may, if he wishes, bypass either one or even bypass

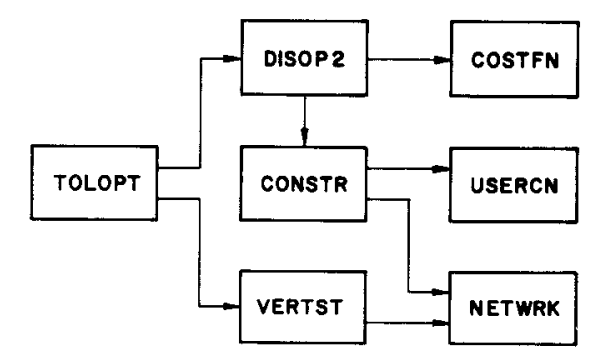

Fig. 4. The overall structure of ToLopt. The user is responsible for NETWRK and USERCN. 
TABLE II

Summary of Features, Options, Parameters, and Subroutines Required

\begin{tabular}{|c|c|c|c|}
\hline Features & Type & Options & Parameters ${ }^{\dagger} /$ subroutines \\
\hline Design parameters & Nominal and tolerance & $\begin{array}{l}\text { Variable or fixed } \\
\text { Relative or absolute } \\
\text { tolerances }\end{array}$ & $\begin{array}{l}\text { Number of parameters } \\
\text { Starting values } \\
\text { Indication for fixed or variable } \\
\text { parameters and relative or absolute } \\
\text { tolerances }\end{array}$ \\
\hline \multirow[t]{2}{*}{$\begin{array}{l}\text { Objective } \\
\text { function }\end{array}$} & Cost & $\begin{array}{l}\text { Reciprocal of } \\
\text { relative and/or absol- } \\
\text { ute tolerances }\end{array}$ & Weighting factors \\
\hline & & other & $\begin{array}{l}\text { Subroutine to define the objective } \\
\text { function and its partial derivatives }\end{array}$ \\
\hline $\begin{array}{l}\text { Vertices } \\
\text { selection* }\end{array}$ & $\begin{array}{l}\text { Gradient direction } \\
\text { strategy }\end{array}$ & & $\begin{array}{l}\text { Maximum allowable number of calls } \\
\text { of the vertices selection subroutine }\end{array}$ \\
\hline \multirow[t]{3}{*}{ Constraints } & $\begin{array}{l}\text { Specifications on } \\
\text { functions of } \\
\text { network parameters }\end{array}$ & Upper and/or lower & $\begin{array}{l}\text { Sample points (e.g., frequency) } \\
\text { Specifications } \\
\text { Subroutine to calculate, for example, } \\
\text { the network response and its partial } \\
\text { derivatives (NETWR) }\end{array}$ \\
\hline & $\begin{array}{l}\text { Network parameter } \\
\text { bounds }\end{array}$ & & Upper and lower bounds \\
\hline & Other constraints & As many as required & $\begin{array}{l}\text { Subroutine to define the constraint } \\
\text { functions and their partial de- } \\
\text { rivatives (USERCN) }\end{array}$ \\
\hline \multirow[t]{2}{*}{$\begin{array}{l}\text { Non1inear } \\
\text { programming }\end{array}$} & $\begin{array}{l}\text { Bandler-Charalambous } \\
\text { minimax }\end{array}$ & $\begin{array}{l}\text { Least pth optimization } \\
\text { algorithms } \\
\text { See Table I }\end{array}$ & $\begin{array}{l}\text { Controling parameter } \alpha \\
\text { Value(s) of } p \\
\text { Test quantities for termination }\end{array}$ \\
\hline & Exterior-point & & $\begin{array}{l}\text { Optimistic estimate of objective } \\
\text { function } \\
\text { Value of } p\end{array}$ \\
\hline $\begin{array}{l}\text { Solution feasibility } \\
\text { check* }\end{array}$ & Least pth & $\begin{array}{l}\text { Discrete problem } \\
\text { Continuous and discrete } \\
\text { problem }\end{array}$ & $\begin{array}{l}\text { Constraint violation tolerance } \\
\text { Value of } p\end{array}$ \\
\hline $\begin{array}{l}\text { Unconstrained } \\
\text { minimization } \\
\text { method }\end{array}$ & Quasi-Newton & $\begin{array}{l}\text { Gradient checking at } \\
\text { starting point by } \\
\text { numerical perturbation }\end{array}$ & $\begin{array}{l}\text { Number of function evaluations } \\
\text { allowed } \\
\text { Estimate of lower bound on least pth } \\
\text { objective } \\
\text { Test quantities for termination }\end{array}$ \\
\hline $\begin{array}{l}\text { Discrete } \\
\text { optimization* }\end{array}$ & Dakin tree-search & $\begin{array}{l}\text { Reduction of dimen- } \\
\text { sionality } \\
\text { User supplied or program } \\
\text { determined initial upper } \\
\text { bound on objective func- } \\
\text { tion } \\
\text { Single or multiple } \\
\text { optimum discrete solu- } \\
\text { tion } \\
\text { Unzform or nonuniform } \\
\text { quantization step sizes }\end{array}$ & $\begin{array}{l}\text { Upper bound on objective function } \\
\text { Maximum permissible number of nodes } \\
\text { Discrete values on step sizes } \\
\text { Number of discrete variables } \\
\text { Discrete value tolerance } \\
\text { Order of partitioning } \\
\text { Indication for discrete variables }\end{array}$ \\
\hline
\end{tabular}

† Parameters associated with the options are not explicitly listed.

* These features are optional and may be bypassed.

the whole routine by supplying his own vertices, or set up his own strategy of vertices selection routine.

The user supplies three sets of numbers, the elements of which correspond to the controlling parameter $\psi_{i}$, the specification $S_{i}$, and the weighting factor $w_{i} . \psi_{i}$ is an independent parameter, e.g., frequency, or any number to identify a particular function. $w_{i}$ is given by

$$
w_{i}= \begin{cases}+1 & \text { if } S_{i} \text { is an upper specification } \\ -1 & \text { if } S_{i} \text { is a lower specification. }\end{cases}
$$

If both upper and lower specifications are assigned to one point, the user can treat it as two points, one with an upper specification and the other with a lower specification. The theory presented earlier will apply in this case under the monotonicity restrictions.

The scheme will, for each $i$, select a set of appropriate $\mu$. Corresponding to each $\boldsymbol{\mu}$, the values $\psi_{i}, \aleph_{i}$, and $w_{i}$ are stored. This information is outputted and used for forming the constraint functions.

The constraints associated with response specifications are of the form

$$
g=w(S-F) \geq 0
$$

with appropriate subscripts, where $F$ is the circuit response function of $\phi$ and $\psi$, and $w$ and $S$ are as before. 
The parameter constraints are

$$
\phi_{j}{ }^{0}-\epsilon_{j}-\phi_{l j} \geq 0
$$

and

$$
\phi_{u j}-\phi_{j}{ }^{0}-\epsilon_{j} \geq 0
$$

where $\phi_{u j}$ and $\phi_{l y}, j \in I_{\phi}$ are the user-supplied upper and lower bounds.

\section{Updating Procedure}

Once the constraints have been selected, optimization is started with a small value of $p$ and $\alpha(p=\alpha=10$ as default values). We have decided to call the routine for updating constraints whenever the $\alpha$ value is updated or the optimization with the initial value of $p$ is completed, until the maximum number of calls is exceeded, or when there is no change of values for consecutive calls. For updating the values, we add new values of $\mu$ to the existing ones without any eliminations. This may not be the most efficient way, but it will be stable.

\section{EXAMPLES}

\section{Example 1}

To illustrate the basic ideas of different cost functions, variable nominal point, and continuous and discrete solutions, a two-section 10:1 quarter-wave transformer is considered [23]. Table III shows the specifications of the design and the result of a minimax solution without tolerances. Fig. 5 shows the contours of $\max _{i}\left|\rho_{i}\right|$ over the range of sample points. The region $R_{c}$ satisfies all the assumptions. Two cost functions, namely, $C_{1}=1 / t_{Z_{1}}+$ $1 / t_{Z_{2}}$ and $C_{2}=1 / \epsilon_{Z_{1}}+1 / \epsilon_{Z_{2}}$ are optimized for the continuous case. The optimal solution with a fixed nominal point at a yields a continuous tolerance set of 8.3 percent and 7.7 percent for $C_{1}$. For the same function with a variable nominal point, the set is $\{12.8,12.8\}$ percent with nominal solution at $\mathbf{b}$. The tolerance set for $C_{2}$ is $\{15.0,9.1\}$ percent with nominal solution at $\mathbf{c}$. $\mathbf{d}$ and $\mathbf{e}$ correspond to the two discrete solutions with tolerance $\mathbf{1 0}$ percent and 15 percent. This example depicts an important fact that an optimal discrete solution cannot always be obtained by rounding or truncating the continuous tolerances to the discrete values. The nominal points must be allowed to move.

\section{Example $\mathscr{2}$}

To illustrate the branch and bound strategy, a 3-component $L C$ low-pass filter is studied [12]. The circuit is shown in Fig. 6. Table IV summarizes the specifications and Table $\mathrm{V}$ lists the results for both the continuous and the discrete solutions. Two different tree structures are shown in Figs. 7 and 8 . This example illustrates that the tree structure, and hence the computational effort, is dependent upon the order of partitioning on the discrete variables. An asterisk attached to the node denotes an optimum discrete solution. It may be noted that one of the discrete solutions, as well as the continuous solution,
TABLE III

Two-Section 10:1 Quarter-Wave Transformer

\begin{tabular}{llcl}
\hline $\begin{array}{l}\text { Relative } \\
\text { Bandwidth }\end{array}$ & $\begin{array}{l}\text { Sample } \\
\text { Points } \\
(\mathrm{GHz})\end{array}$ & $\begin{array}{c}\text { Reflection Coefficient } \\
\text { Specification }\end{array}$ & Type \\
\hline $100 \%$ & $0.5,0.6, \ldots, 1.5$ & 0.55 & upper \\
\hline & Minimax solution (no tolerances) & $|0|=0.4286$ & \\
\hline
\end{tabular}

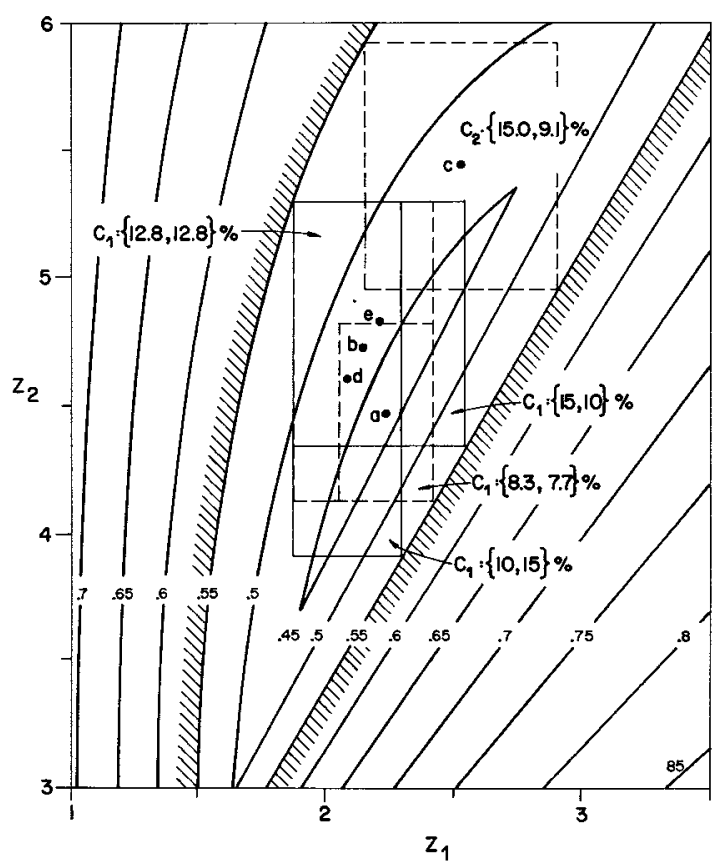

Fig. 5. Contours of $\max \left|\rho_{i}\right|$ with respect to $Z_{1}$ and $Z_{2}$ for example 1 indicating a number of relevant solution points (see text).

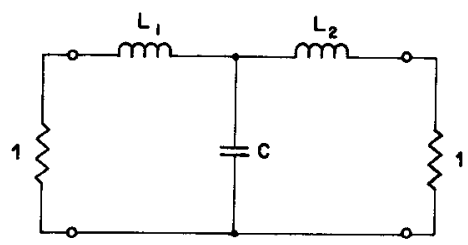

Fig. 6. The circuit for example 2 .

yields symmetrical results, although symmetry is not assumed in the formulation of the problem.

\section{Example 8}

Consider a five-section cascaded transmission-line lowpass filter with characteristic impedances fixed at the values

$$
\begin{array}{r}
Z_{1}{ }^{0}=Z_{3}{ }^{0}=Z_{5}{ }^{0}=0.2 \\
Z_{2}{ }^{0}=Z_{4}{ }^{0}=5.0
\end{array}
$$

and terminated in unity resistances $[1],[6]$. See Table VI for the specifications. The length units are normalized with respect to $l_{q}=c / 4 f_{0}$, where $f_{0}=1 \mathrm{GHz}$. Two problems are presented here.

1) A uniform 1-percent relative tolerance is allowed for each impedance. Maximize the absolute tolerances on the 
TABLE IV

LC Low-Pass FrLter

\begin{tabular}{lccc}
\hline $\begin{array}{c}\text { Frequency } \\
\text { Range } \\
(\mathrm{rad} / \mathrm{s})\end{array}$ & $\begin{array}{c}\text { Sample } \\
\text { Points } \\
\text { (rad/s) }\end{array}$ & $\begin{array}{c}\text { Insertion Loss } \\
\text { Specification } \\
\text { (dB) }\end{array}$ & Type \\
\hline $\begin{array}{c}0-1 \\
2.5\end{array}$ & $0.5,0.55,0.6,1.0$ & 1.5 & upper (passband) \\
& 2.5 & 25 & 10wer (stopband) \\
& Minimax solution (no tolerances) \\
& passband $0.53 \mathrm{~dB}$ \\
stopband 26 & $\mathrm{~dB}$ & \\
\hline
\end{tabular}

TABLE V

$L C$ Low-Pass Filiter Tolerance Optimization $\left(C_{1}\right)$

\begin{tabular}{|c|c|c|c|c|c|}
\hline \multirow[t]{2}{*}{ Parameters } & \multicolumn{2}{|c|}{ Continuous Solution } & \multicolumn{3}{|c|}{$\begin{array}{l}\text { Discrete Solution } \\
\text { From }\{1,2,5,10,15\} \%\end{array}$} \\
\hline & & & 1 & 2 & 3 \\
\hline$x_{2}=t_{L_{1}}$ & $3.5 \%$ & $9.9 \%$ & $5 \%$ & $10 \%$ & $10 \%$ \\
\hline$x_{1}=t_{C}$ & $3.2 \%$ & $7.6 \%$ & $10 \%$ & $5 \%$ & $10 \%$ \\
\hline$x_{3}=t_{L_{2}}$ & $3.5 \%$ & $9.9 \%$ & $10 \%$ & $10 \%$ & $5 \%$ \\
\hline$x_{5}=L_{1}^{0}$ & 1.628 & & 1.999 & & \\
\hline$x_{4}=c^{0}$ & 1.090 & & 0.906 & & \\
\hline$x_{6}=L_{2}^{0}$ & 1.628 & & 1.999 & & \\
\hline
\end{tabular}

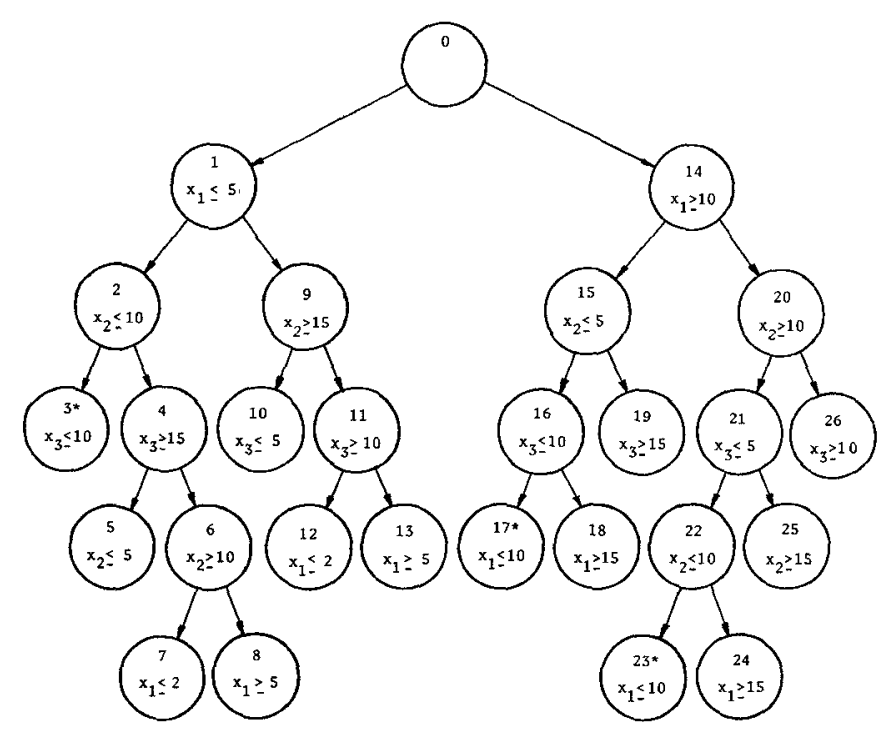

Fig. 7. Tree structure for example 2, partitioning on $x_{1}$ first (see Table V). Asterisk denotes optimal discrete solutions.

section lengths and find the corresponding nominal lengths. Let the cost function be

$$
C_{2}=\sum_{i=1}^{5} \frac{1}{\epsilon_{l_{i}}}
$$

2) A uniform length tolerance of 0.001 is given. Maximize the relative tolerances on the impedances and obtain the corresponding nominal lengths. Let the cost function be

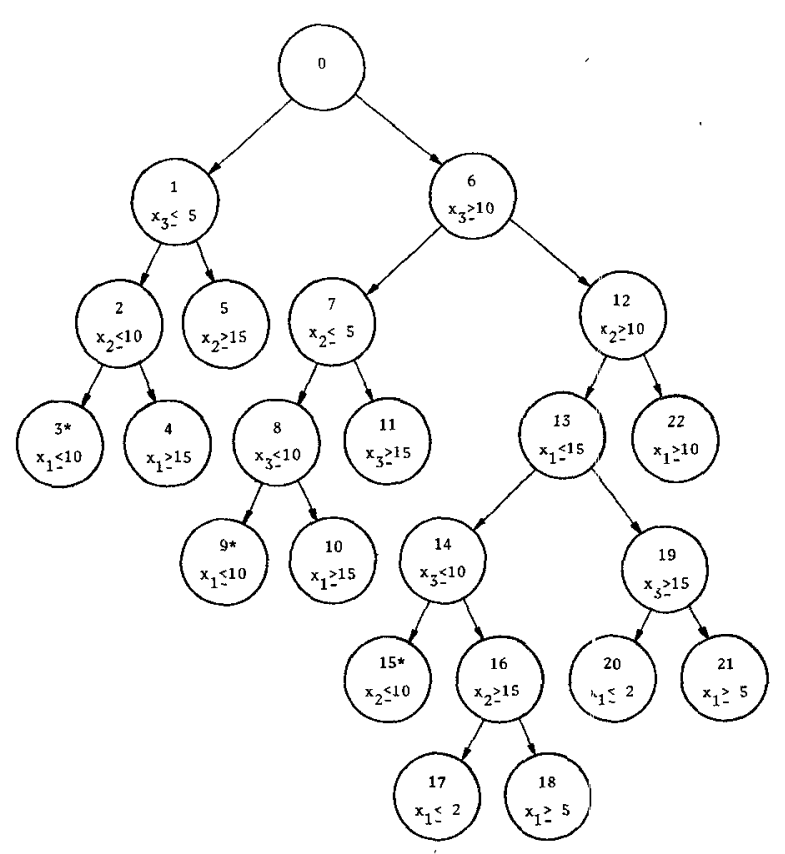

Fig. 8. Tree structure for example 2 , partitioning on $x_{3}$ first (see Table V). Asterisk denotes optimal discrete solutions.

$$
C_{1}=\sum_{i=1}^{5} \frac{1}{t_{Z_{i}}} .
$$

The filter has 10 circuit parameters which may be arranged in the order $Z_{1}, Z_{2}, \cdots, Z_{5}, l_{1}, l_{2}, \cdots, l_{5}$. To simplify the problem, symmetry with respect to a center line 
TABLE VI

Five-Section Transmission-Line Low-Pass Filiter

\begin{tabular}{llll}
\hline $\begin{array}{l}\text { Frequency } \\
\text { Range } \\
\text { (GHz) }\end{array}$ & $\begin{array}{l}\text { Sample } \\
\text { Points } \\
\text { (GHz) }\end{array}$ & $\begin{array}{c}\text { Insertion Loss } \\
\text { Specification } \\
\text { (dB) }\end{array}$ & Type \\
\hline $0-1$ & $.35, .4, .45, .75, .8, .85,1.0$ & .02 & upper (passband) \\
$2.5-10$ & $2.5,10$ & 25 & lower (stopband) \\
\hline
\end{tabular}

through the circuit is assumed. The matrix $\mathbf{S}$ is given by

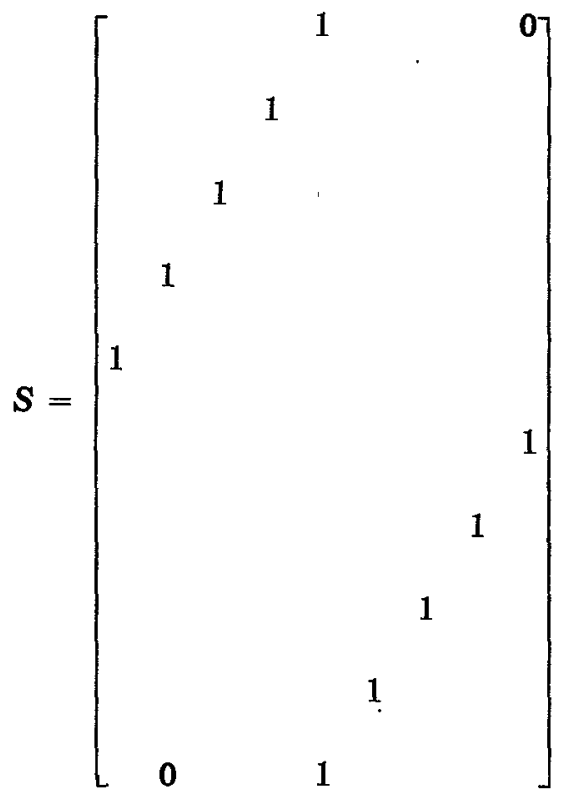

which also implies that $l_{1}^{0}=l_{5}^{0}$, and $l_{2}^{0}=l_{4}^{0}$. The same kind of equalities is applied to the tolerances.

The first vertices elimination scheme is applied with values at the optimal nominal values without tolerances, and the relative impedance tolerance and the absolute length tolerances at 2 percent and 0.002 , respectively. A total of 46 vertices corresponding to all the frequency points were selected from a possible set of $9 \times 2^{10}$. Fourteen were further eliminated by symmetry. A final total of 15 constraints were chosen after comparing relative magnitudes. These 15 constraints were used throughout the optimization. The continuous and discrete solutions to the two problems are shown in Tables VII and VIII.

\section{DISCUSSION AND CONCLUSIONS}

We have described an efficient user-oriented program for circuit design with worst case tolerance considerations embodying a number of new ideas and recent algorithms. The automated scheme could start from an arbitrary initial acceptable or unacceptable design to obtain continuous and/or discrete optimal nominal parameter values and tolerances simultaneously. However, optimization of the nominal values without tolerances should preferably be done first to obtain a suitable starting point. The effort is small compared with the complete tolexance problem when a small value of $p$ greater than unity, e.g., $p=2$, is
TABLE VII

Five-Stectron Transmission-Line Low-Pass Filter Tolerance Optimization $\left(C_{1}\right)$

\begin{tabular}{lcc}
\hline Parameters & Continuous Solution & $\begin{array}{c}\text { Discrete Solution } \\
\text { From }(.5,1,1.5,2,3,5\} \%\end{array}$ \\
\hline$t_{z_{1}}=t_{z_{5}}$ & $3.56 \%$ & $3 \%$ \\
$t_{z_{2}}=t_{z_{4}}$ & $2.27 \%$ \\
$t_{z_{3}}$ & 0.0786 \\
$\ell_{1}^{0}=\ell_{5}^{0}$ & 0.1415 \\
$\ell_{2}^{0}=\ell_{4}^{0}$ & 0.1736 \\
$\ell_{3}^{0}$ & $z_{1}^{0}=z_{3}^{0}=z_{5}^{0}=0.2, z_{2}^{0}=z_{4}^{0}=5$ \\
& $\varepsilon_{\ell_{i}}=0.001, \quad i=1,2, \ldots, 5$ \\
&
\end{tabular}

TABLE VIII

Five-Section Transmission-Line Low-Pass Filter Tolerance Optimization $\left(C_{2}\right)$

\begin{tabular}{|c|c|c|}
\hline Parameters & Continuous Solution & $\begin{array}{l}\text { Discrete Solution } \\
.0005 \text { Step Size }\end{array}$ \\
\hline$\varepsilon_{\ell_{1}}=\varepsilon_{\ell_{5}}$ & 0.0033 & 0.0030 \\
\hline$\varepsilon_{\ell_{2}}=\varepsilon_{\ell_{4}}$ & 0.0028 & 0.0030 \\
\hline${ }^{\varepsilon} \ell_{3}$ & 0.0027 & 0.0025 \\
\hline$\ell_{1}^{0}=\ell_{5}^{0}$ & \multicolumn{2}{|c|}{0.0788} \\
\hline$\ell_{2}^{0}=\ell_{4}^{0}$ & \multicolumn{2}{|c|}{0.1414} \\
\hline$\ell_{3}^{0}$ & \multicolumn{2}{|c|}{0.1738} \\
\hline \multicolumn{3}{|c|}{$z_{1}^{0}=z_{3}^{0}=z_{5}^{0}=0.2, z_{2}^{0}=z_{4}^{0}=5$} \\
\hline
\end{tabular}

used. An exact minimax solution is not needed. This also serves as a feasibility check. If $R_{c}$ is indicated to be empty, the designer has to relax some specifications or change his circuit. The solution process may also provide valuable information to the designer, e.g., parameter or frequency symmetry.

The problem without tolerances may be solved easily by available programs such as CANOPT [24]. The user may alternatively utilize the optimization part, namely DIsop2, of the present package.

It is good practice first to obtain a continuous solution before attempting the discrete problem. A useful feature of the program is that, for example, depending on information obtained from prior runs, the user can reenter at a number of different stages of the solution process.

The assumptions on the constraints may be difficult to 
test. For this reason, a Monte Carlo simulation of the final solution is usually carried out.

We have presented results for two basic types of cost function. A more realistic cost-tolerance model should be established from known component cost data, if these are unsuitable in particular cases.

The complete Fortran listing and documentation for TOLOPT will be made available [25]. It is very important that the user-provided routine for network function computation and the respective sensitivities be efficient. Typical running time for a small and medium size problem (less than 10 network parameters or 20 optimization parameters) will be from 2 to $20 \mathrm{~min}$. The execution time on a CDC 6400, taking the $L C$ low-pass filter as an example, was less than $10 \mathrm{~s}$ for the continuous case, and a total of 80-100 s for the entire problem, depending on the order of partitioning. The five-section transmission-line example needed about $300-400 \mathrm{~s}$.

\section{REFERENCES}

[1] J. W. Bandler, P. C. Liu, and J. H. K. Chen, "Computer-aided tolerance optimization applied to microwave eircuits," in IEEE Int. Microwave Symp. Dig. (Atlanta, Ga., June 1974), pp. 275-277.

[2] J. W. Bandler, B. L. Bardakjian, and J. H. K. Chen, "Design of recursive digital filters with optimum word length coefficients," presented at the 8th Princeton Conf. Information Sciences and Systems, Princeton, N. J., Mar. 1974.

[3] a) J. W. Bandler and J. H. K. Chen, "DISOPT-a general program for continuous and discrete nonlinear programming problems," Int. J. Syst. Sci., to be published.

b) J. H. K. Chen, McMaster Univ., Hamilton, Ont., Canada, Int. Rep. Simulation, Optimization, and Control SOC-29, Mar. 1974.

[4] R. J. Dakin, "A tree-search algorithm for mixed integer pro-

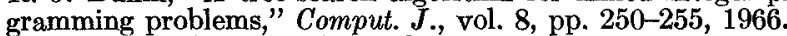

[5] R. Fletcher, "FORTRAN subroutines for minimization by quasi-Newton methods," Atomic Energy Research Establishment, Harwell, Berks., England, Rep. AERE-R7125, 1972.

[6] J. W. Bandler and C. Charalambous, "Practical least $p$ th optimization of networks," IEEE Trans. Microwave Theory Tech. (1972 Symp. Issue), vol. MTT-20, pp. 834-840, Dec. 1972.

[7] C. Charalambous and J. W. Bandler, "New algorithms for network optimization," IEEE Trans. Microwave Theory Tech. (1973 Symp. Issue), vol. MTT-21, pp. 815-818, Dec. 1973.

[8] J. W. Bandler and C. Charalambous, "Nonlinear programming using minimax techniques," J. Optimiz. Theory Appl., vol. 13, pp. 607-619, June 1974 .

[9] C. Charalambous, "A unified review of optinaization," IEEE Trans. Microwave Theory Tech. (Special Issue on ComputerOriented Microwave Practices), vol. MTT-22, pp. 289-300, Mar. 1974.

[10] W. Y. Chu, "Extrapolation in least $p$ th approximation and nonlinear programming," McMaster Univ., Hamilton, Ont., Canada, Int. Rep. Simulation, Optimization, and Control, SOC-71, Dec. 1974 .

[11] J. W. Bandler, "Optimization of design tolerances using nonlinear programming," J. Optimiz. Theory Appl., vol. 14, pp. 99-114, 1974

[12] J. W. Bandler and P. C. Liu, "Automated network design with optimal tolerances," IEEE Trans. Circuits Syst., vol. CAS-21, pp. 219-222, Mar. 1974.

[13] J. F. Pinel and K. A. Roberts, "Tolerance assignment in linear networks using nonlinear programming," IEUE Trans. Circuit Theory, vol. CT-19, pp. 475-479, Sept. 1972 .

[14] E. M. Butler, "Realistic design using large-change sensitivities and performance contours," IEEE Trans. Circuit Theory (Special Issue on Computer-Aided Circuit Design), vol. CT-18, pp. 58-66, Jan. 1971.

[15] B. J. Karafin, "The optimum assignment of component tolerances for electrical networks," Bell Syst. Tech. J., vol. 50, pp. 1225-1242, Apr. 1971.

[16] O. L. Mangasarian, Nonlinear Programming. New York: McGraw-Hill, 1969.

[17] J. W. Bandler and P. C. Liu, "Some implications of biquadratic functions in the tolerance problem," in Proc. IEEE Int. Symp. Circuits and Systems (San Francisco, Calif., Apr. 1974), pp. $740-744$.

[18] P. Lancaster, Theory of Matrices. New York: Academic, 1969.

[19] A. V. Fiacco and G. P. McCormick, Nontinear Programming: Sequential Unconstrained Minimization Techniques. New York: Wiley, 1968

[20] F. A. Lootsma, "A survey of methods for solving constrained minimization problems via unconstrained minimization," in Numerical Methods for Nonlinear Optimization, F. A. Lootsma, Ed. New York: Academic, 1972.

[21] P. E. Gill and W. Murray, "Quasi-Newton methods for unconstrained optimization," J. Inst. Math. Its Appl., vol. 9, pp. 91-108, 1972.

[22] R. Fletcher, "A new approach to variable metric algorithms," Comput. J., vol. 13, pp. 317-322, 1970.

[23] J. W. Bandler and P. A. Macdonald, "Cascaded noncommensurate transmission-line networks as optimization problems," IEEE Trans. Circuit Theory (Corresp.), vol. CT-16, pp. 391-394, Aug. 1969.

[24] J. W. Bandler, J. R. Popović, and V. K. Jha, "Cascaded network optimization program," IEEE Trans, Microwave Theory Tech. (Special Issue on Computer-Oriented Microwave Practices), vol. MTT-22, pp. 300-308, Mar. 1974.

[25] J. W. Bandler, J. H. K. Chen, P. Dalsgaard, and P. C. Liu, "TOLOPT-A program for optimal, continuous or discrete, design centering and tolerancing," McMaster Univ., Hamilton, Ont., Canada, Int. Rep. Simulation, Optimization, and Control. 